febrile convulsion," distinct from the epilepsies. (Millichap JG. Definition of febrile seizures. In: Febrile Seizures ed Nelson KB, Ellenberg JH, New York; Raven Press, 1981;1-3). Genetic studies show that inheritance of FS is polygenic with no significant differences between simple and complex FS patients. The cytokine gene ILIB-511 is associated with sporadic but not familial simple FS. Cytokine genes may act as enhancers or attenuators of FS susceptibility. Genetic association studies may lead to a better understanding of the molecular basis of FS and the relation to epilepsy. (Kira R et al. Genetic susceptibility to febrile seizures. Brain Dev Jan 2010;32:57-63).

\title{
PERIPHERAL NEUROPATHIES
}

\section{PHARMACOKINETICS OF INTRAVENOUS IMMUNOGLOBULIN AND OUTCOME OF GUILLAIN-BARRE SYNDROME}

The relation of pharmacokinetics of intravenous immunoglobulin (IVIg) to the outcome of Guillain-Barre syndrome (GBS) was determined in 174 adults treated at Erasmus Medical Center, Rotterdam, The Netherlands. All patients were unable to walk unaided and all received the standard dose IVIg, $0.4 \mathrm{~g} / \mathrm{kg} / \mathrm{body}$ weight $/$ per day for 5 consecutive days (total $2 \mathrm{~g} / \mathrm{kg} /$ body weight), within 2 weeks of onset of weakness. Total IgG levels in serum samples obtained immediately before and 2 weeks after the start of IVIg administration were determined by turbidimetry and related to clinical outcome at 6 months. The increase in serum IgG 2 weeks after IVIg varied widely between patients (mean $7.8 \mathrm{~g} / \mathrm{L}, \mathrm{SD} 5.6 \mathrm{~g} / \mathrm{L}$ ). Patients with a low $\mathrm{IgG}$ recovered significantly more slowly, and fewer could walk unaided at 6 months $(\mathrm{p}<0.001)$. The association of low $\mathrm{IgG}$ with poor outcome was independent of other known prognostic factors $(\mathrm{p}=0.022)$. Patients with a small increase in serum IgG may benefit from a higher dosage or second course of IVIg. (Kuitwaard K, de Gelder J, Tio-Gillen AP, et al. Pharmacokinetics of intravenous immunoglobulin and outcome in Guillain-Barre syndrome. Ann Neurol Nov 2009;66:597-603). (Respond: Dr K Kuitwaard, Department of Neurology, Room H 673, Erasmus Medical Center, PO Box 2040, 3000 CA Rotterdam, The Netherlands. E-mail: k.kuitwaard@erasmusmc.nl).

COMMENT. The high variability of IVIg pharmacokinetics in patients with GBS is related to clinical course and outcome. Monitoring the serum IgG levels may optimize the use of IVIg treatment in individual GBS patients.

In an editorial, Cornblath DR and Hughes RAC (Ann Neurol 2009;66:569-570) comment that despite the benefits of treatment of GBS with IVIg or plasma exchange, 2$3 \%$ of patients with severe GBS die within 1 year and $15-18 \%$ have significant disability. The IVIg dose was chosen based initially on studies in idiopathic thrombocytopenia, and higher doses or longer courses have not been properly evaluated in GBS. Furthermore, not all cases are acute inflammatory demyelinating polyneuropathy. Some cases are acute motor axonal neuropathy and Fisher's syndrome that may respond differently. Controlled clinical trials show benefit of IVIg in CIDP, GBS, and multifocal neuropathy. Trials of IVIg in different doses and duration are also indicated in other diseases. 